\title{
CARACTERÍSTICAS FISIOLÓGICAS E DE DESENVOLVIMENTO DE Cyperus difformis L. RESISTENTE E SUSCETÍVEL AO HERBICIDA PYRAZOSULFURON-ETHYL
}

\section{PHYSIOLOGIC CHARACTERIZATICS AND DEVELOPMENT OF RESISTANT AND SUSCEPTIBLE Cyperus difformis L. TO PYRAZOSULFURON-ETHYL HERBICIDE}

\author{
Taísa DAL MAGRO ${ }^{1}$ \\ Dirceu AGOSTINETTO \\ Antônio Alberto da SILVA ${ }^{3}$ \\ Leandro VARGAS ${ }^{4}$ \\ Luís Eduardo PANOZZO 5 \\ Leandro GALON ${ }^{6}$
}

\section{RESUMO}

Os biótipos de plantas daninhas resistentes a herbicidas podem apresentar diferenças fisiológicas e de desenvolvimento quando comparado a biótipos suscetíveis. O objetivo deste trabalho foi comparar características fisiológicas e de desenvolvimento de biótipos Cyperus difformis resistente e suscetível ao herbicida pyrazosulfuron-ethyl. Foram conduzidos dois experimentos em casa de vegetação, um na Universidade Federal de Pelotas (Exp. I) e outro na Universidade Federal de Viçosa (Exp. II), em delineamento experimental completamente casualizado, com quatro repetições. No primeiro experimento (Exp. I) os tratamentos constaram de biótipos de $C$. difformis resistente (CYPDI 9) e suscetível (CYPDI 8) ao herbicida pyrazosulfuron-ethyl e de seis épocas de coletas (14, 28, 42, 56, 70 e 84 dias após a emergência) e no segundo (Exp. II) os biótipos de C. difformis resistente e suscetível foram avaliados com e sem a aplicação do herbicida pyrazosulfuronethyl, na dose de $20 \mathrm{~g} \mathrm{ha}^{-1}$. As variáveis avaliadas no Exp. I foram área foliar (AF), massa da parte aérea seca (MPAS), taxa de crescimento absoluto e a relação AF e MPAS; no Exp. II, $\mathrm{CO}_{2}$ consumido, taxa de fluxo de gases, concentração de $\mathrm{CO}_{2}$ subestomático, taxa transpiratória, condutância estomática de vapores de água, taxa fotossintética e eficiência do uso da água. Observou-se que os biótipos de $C$. difformis resistente e suscetível ao herbicida pyrazosulfuron-ethyl apresentam características fisiológicas e de desenvolvimento semelhantes.

Palavras-chave: arroz irrigado; junquinho; resistência.

\section{ABSTRACT}

The resistance of weed to herbicides can cause alterations in physiologic characteristics and development of the resistant biotype. The objectives of this work went to compare physiologic characteristics and development from biotypes of resistant and susceptible $C$. difformis to ALS inhibiting herbicides. For that, two Studies were driven at green house of UFPel (Study I) and of UFV (Study II) in completely randomized design, with four replicates. In Study I the treatments consisted of resistant biotype (CYPDI 9) and susceptible (CYPDI 8) of C. difformis to herbicide pyrazosulfuron-ethyl and six times of collections $(14,28,42,56,70$ and 84 days after the emergency) and in Study II the same biotypes (resistant and susceptible) added of the application of pyrazosulfuron-ethyl in the rate of $20 \mathrm{~g} \mathrm{ha}^{-1}$. The variable availed were leaf area, aerial dry mass, rate growth and the relation leaf area and aerial dry mass, in Study I and consumed $\mathrm{CO}_{2}$, rate of flow of gases for the stomata, concentration of $\mathrm{CO}_{2}$ sub-stomatal, transpiration rate, stomatic conductance of steams of water, photosynthetic rate and efficiency of the water use in Study II. Before the results, it can be observed that the resistant and susceptible biotypes of $C$. difformis to pyrazosulfuron-ethyl herbicide, present physiologic characteristics and development similar.

Key-words: rice irrigated; smallflower umbrella sedge; resistance.

\footnotetext{
1 Engenheira Agrônoma, Doutoranda do Programa de Pós-Graduação em Fitossanidade da Faculdade de Agronomia Eliseu Maciel (FAEM) da Universidade Federal de Pelotas (UFPel) e Professora do Curso de Agronomia da Universidade de Caxias do Sul (UCS). Rua Farroupilha n¹80 apartamento 203, Centro, Vacaria - RS, CEP 95200-000. E-mail: taisadm@yahoo.com.br;

2 Engenheiro Agrônomo, Professor do Departamento de Fitossanidade, FAEM/UFPel. Bolsista em produtividade do CNPq. E-mail: agostinetto@ig.com.br:

3 Engenheiro Agrônomo, Professor do Departamento de Fitotecnia da Universidade Federal de Viçosa (UFV). E-mail: aasilva@ufv.br;

${ }^{4}$ Engenheiro Agrônomo, Pesquisador da Embrapa CNPT e Professor do Programa de Pós-Graduação em Fitossanidade, FAEM/UFPel. E-mail: vargas@cnpt.embrapa.br

${ }^{5}$ Engenheiro Agrônomo, Mestrando do Programa de Pós-Graduação em Fitossanidade, FAEM/UFPel. E-mail: lepanozzo@gmail.com

${ }^{5}$ Engenheiro Agrônomo, Mestrando do Programa de Pós-Graduação em Fitossanidade, FAEM/UFPel. E-mail: lepanozzo@gan
${ }^{5}$ Engenheiro Agrônomo, Doutorando do Programa de Pós-Graduação em Fitotecnia, UFV. E-mail: galonleandro@ig.com.br
} 
DAL MAGRO, T. et al. Características fisiológicas e de desenvolvimento...

\section{INTRODUÇÃO}

O gênero Cyperus inclui-se entre as principais plantas daninhas que infestam as lavouras de arroz irrigado, sendo seu controle realizado principalmente por herbicidas inibidores da enzima acetolactato sintase (ALS). O uso continuado de herbicidas com mesmo mecanismo de ação selecionou biótipos de Cyperus difformis L. (junquinho) resistente aos herbicidas inibidores da enzima ALS no Estado de Santa Catarina (Noldin et al., 2002).

Estudos de crescimento de plantas frequentemente são utilizados para conhecer a ecologia de espécies e para análise comparativa entre plantas daninhas e culturas, podendo também ser aplicado para estudos de plantas daninhas resistentes a herbicidas (Rampelotti et al., 2003; Vargas et al., 2005). Neste contexto, o conhecimento das características biológicas entre biótipos resistentes e suscetíveis torna-se importante na definição de sua capacidade competitiva, podendo com isso auxiliar nos métodos de manejo adotados. Estudos de interferência de plantas daninhas, geralmente, consideram apenas características morfológicas das espécies (Agostinetto et al., 2008; Fleck et al., 2008). No entanto, estes resultados acrescidos da caracterização fisiológica, poderão proporcionar maior entendimento e elucidação dos fenômenos ocorridos, como a eficiência no uso da água (Concenço et al., 2007), de nutrientes e da radiação solar.

Plantas mais eficientes no uso da água produzem maior quantidade de massa da parte aérea seca por grama de água transpirada. Para o biótipo de azevém suscetível a herbicidas inibidores da enzima 5-enolpiruvilshiquimato-3-fosfato sintase (EPSPs), observou-se maior produção de massa da parte aérea seca e incorporação de $\mathrm{CO}_{2}$, comparativamente ao resistente (Concenço et al., 2007).

O entendimento dos mecanismos de adaptação ao ambiente por biótipos resistentes pode ser usado no desenvolvimento de ferramentas no controle da resistência. A literatura relata que biótipo de Lolium multiflorum suscetível ao glyphosate (Vargas et al., 2005) e Lactuca serriola suscetível a sulfoniluréias (Alcocer-Ruthling et al., 1992), possuem maior capacidade de acúmulo de massa da parte aérea seca, enquanto biótipos de Amaranthus retroflexus (Conard \& Radosevich, 1979) e Chenopodium album (Parks et al., 1996), suscetíveis as triazinas, apresentaram maior área foliar que os biótipos resistentes. Por outro lado, biótipos de Amaranthus powelli resistente a herbicidas inibidores da enzima ALS tiveram redução em seu crescimento inicial, desenvolvimento e valor adaptativo decorrente da resistência (Tardif et al., 2006). Porém, não foram detectadas diferenças na capacidade competitiva entre biótipos de Sagitaria montevidensis resistente a herbicidas inibidores de ALS (Rampelotti et al. 2003) e Abutilon theophrasti resistente às triazinas (Gray et al., 1995). Diante disso, é possível inferir que a ocorrência de alterações fisiológicas e adaptativas em plantas daninhas resultantes de resistência podem ser variadas em função da espécie e do herbicida.

Uma vez estabelecido o problema de resis- tência, há necessidade de adoção de medidas de manejo, as quais poderão ser facilitadas com o conhecimento de características dos biótipos, como competitividade, suscetibilidade a herbicidas e sua adaptabilidade ao ambiente. Assim, o conhecimento das características biológicas dos biótipos é relevante, uma vez que agem diretamente sobre as características morfológicas, as quais definem a capacidade de competir por recursos do meio, como água, luz e nutrientes (Radosevich et al., 1997).

A hipótese da pesquisa foi de que o biótipo de $C$. difformis suscetível ao herbicida pyrazosulfuron-ethyl apresenta superioridade em suas características fisiológicas e de desenvolvimento, comparativamente ao biótipo resistente. Assim, o objetivo do trabalho foi comparar características fisiológicas e de desenvolvimento de biótipos $C$. difformis resistente e suscetível ao herbicida pyrazosulfuron-ethyl.

\section{MATERIAL E MÉTODOS}

Foram conduzidos dois experimentos em casa de vegetação, um na Universidade Federal de Pelotas (UFPel), localizada no Município de Capão do Leão - RS, no período de novembro de 2006 a fevereiro de 2007 (Experimento I) e outro na Universidade Federal de Viçosa (UFV), localizada no Município de Viçosa - MG, no período de março a maio de 2008 (Experimento II). Os tratamentos foram dispostos em delineamento experimental completamente casualizado, com quatro repetições. Cada unidade experimental foi composta por vaso plástico com capacidade para $550 \mathrm{~g}$ de solo, adubado de acordo com a análise do solo. Os vasos foram perfurados ao fundo e colocados em bandejas plásticas contendo água, para que a irrigação ocorresse por capilaridade. As sementes de $C$. difformis resistente (CYPDI 9) e suscetível (CYPDI 8) aos herbicidas inibidores de ALS foram oriundas de populações do Município de Meleiro (49 $33^{\prime} \mathrm{LW}$ e $\left.28^{\circ} 53^{\prime} \mathrm{LS}\right)$ e Itajaí $\left(48^{\circ} 45^{\prime} \mathrm{LW}\right.$ e $\left.26^{\circ} 56^{\prime} \mathrm{LS}\right)$, respectivamente, ambos no Estado de Santa Catarina (SC), identificadas por Noldin et al. (2002), sendo que o biótipo resistente sobreviveu à aplicação de 16 vezes a dose comercial de pyrazosulfuron-ethyl $\left(20 \mathrm{~g} \mathrm{ha}^{-1}\right)$.

\section{Experimento I}

Foi realizado com a finalidade de comparar características de crescimento de biótipos de $C$. difformis resistente e suscetível ao herbicida pyrazosulfuron-ethyl. A semeadura foi realizada em densidades superiores às desejadas, com posterior desbaste para a retirada do excesso de plantas e manutenção de duas plantas por vaso. O solo utilizado foi previamente esterilizado (autoclavado) para evitar a emergência de plantas a partir do banco de sementes.

Os tratamentos constaram de plantas de biótipos resistente e suscetível de $C$. difformis ao herbicida pyrazosulfuron-ethyl e de seis épocas de coletas $(14,28,42,56,70$ e 84 dias após a emergência - DAE).

As variáveis avaliadas, em cada coleta, foram área foliar (AF) e massa da parte aérea seca (MPAS), composta por caule, folhas e estrutura repro- 
dutiva (quando presente). A AF foi determinada com medidor de AF, marca LI-COR, Modelo 3100C. A MPAS foi quantificada pela secagem do material vegetal em estufa, a temperatura de $60{ }^{\circ} \mathrm{C}$, até atingir massa constante.

Foram calculadas a taxa de crescimento absoluto (TCA), em g planta ${ }^{-1}$ dia $^{-1}$, e a relação da AF e MPAS (RAFMAS), em $\mathrm{dm}^{2} \mathrm{~g}^{-1}$ de massa da parte aérea seca. A TCA foi determinada pela razão entre MPAS e número de dias que a planta cresceu, enquanto a RAFMAS foi quantificada pela razão entre a AF e MPAS.

Os dados obtidos foram analisados quanto à sua homocedasticidade e posteriormente submetidos à análise de variância (teste $F)(p \leq 0,05)$. Quando o teste foi significativo, os efeitos de biótipos foram comparados pelo teste $t(p \leq 0,05)$ e os de época de avaliação por modelos de regressão linear $(p \leq 0,05)$, conforme segue:

$$
\begin{aligned}
& y=a+b x \\
& y=a+b x+c x^{2}
\end{aligned}
$$

onde: $a=$ valor mínimo ou máximo estimado para a variável resposta; $b$ e $c=$ inclinação da reta ou curva; $\mathrm{e}, \mathrm{x}=$ dias de crescimento da planta.

\section{Experimento II}

Foi realizado com o intuito de avaliar características fisiológicas dos biótipos de $C$. difformis resistente e suscetível a pyrazosulfuron-ethyl. Para o estabelecimento das populações, a semeadura foi realizada em bandejas plásticas e, aos $15 \mathrm{DAE}$, foi realizado o transplante de uma planta por vaso.

Os tratamentos constaram de plantas de biótipos de $C$. difformis resistente e suscetível ao herbicida pyrazosulfuron-ethyl, com e sem a aplicação do mesmo em dose de $20 \mathrm{~g} \mathrm{ha}^{-1}$ (dose recomendada). A aplicação do herbicida foi realizada em pósemergência, quando as plantas se encontravam com até quatro folhas, utilizando-se pulverizador costal, pressurizado a $\mathrm{CO}_{2}$, equipado com ponta de pulverização do tipo leque 110.015 , calibrado para aplicar $150 \mathrm{dm}^{3} \mathrm{ha}^{-1}$ de calda herbicida.

As variáveis avaliadas foram: $\mathrm{CO}_{2}$ consumido $\left(\Delta \mathrm{C}-\mu \mathrm{mol} \mathrm{mol}{ }^{-1}\right)$; taxa de fluxo de gases pelos estômatos $\left(\mathrm{U}-\mu \mathrm{mol} \mathrm{s}{ }^{-1}\right)$; concentração de $\mathrm{CO}_{2}$ subestomático $\left(\mathrm{C}-\mu \mathrm{mol} \mathrm{mol}^{-1}\right)$; taxa transpiratória $(\mathrm{E}$ -

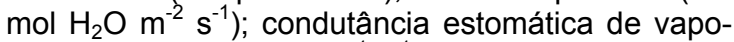
res de água ( $\mathrm{Gs}-\mathrm{mol} \mathrm{m}^{-1} \mathrm{~s}^{-1}$ ); taxa fotossintética ( $A$ - $\mu \mathrm{mol} \mathrm{m}^{-2} \mathrm{~s}^{-1}$ ); e eficiência do uso da água (EUA mol $\mathrm{CO}_{2} \mathrm{~mol} \mathrm{H}_{2} \mathrm{O}^{-1}$ ). As avaliações foram realizadas na folha central da planta, em condições semicontroladas, ou seja, em casa de vegetação aberta, permitindo livre circulação do ar, aos 7, 14 e 21 dias após a aplicação dos tratamentos (DAT), pelo uso de analisador de gases infravermelho (IRGA) marca ADC, modelo LCA 4, previamente calibrado. Um representante de cada tratamento foi avaliado sepa- radamente, para manutenção homogênea das condições ambientais no momento da avaliação (intensidade luminosa, $\mathrm{O}_{2}, \mathrm{CO}_{2} \ldots$ ), que podem oscilar com o decorrer da avaliação em função, principalmente, das alterações de umidade, temperatura e radiação solar. Foi avaliada também, a MPAS, aos 28 DAT, quando o material coletado foi seco em estufa a $60^{\circ} \mathrm{C}$ até atingir massa constante.

Os dados obtidos foram analisados quanto à sua homocedasticidade e posteriormente submetidos à análise de variância (teste $F)(p \leq 0,05)$. Em sendo significativo, os efeitos de biótipo e tratamentos foram avaliados pelo teste $t(p \leq 0,05)$.

\section{Experimento I}

\section{RESULTADOS E DISCUSSÃO}

As variáveis AF, MPAS, TCA e RAFMAS não apresentaram interação dos fatores testados, apresentando apenas efeito de épocas de avaliação (Figuras 1 a 4). Para a variável $A F$, os dados se adequaram ao modelo quadrático, no qual a produção de AF atingiu o ponto de máxima aos $62 \mathrm{DAE}$, sendo observada tendência de decréscimo com o passar do tempo (Figura 1). Entretanto, ao considerar os intervalos de confiança, verificou-se não haver diferença na AF para períodos de avaliação compreendidos entre 42 e 84 DAE.

A produção de MPAS foi crescente com o tempo de sobrevivência das plantas, representada pelo ajuste dos dados ao modelo linear (Figura 2), o qual apresentou acréscimo diário de $0,0049 \mathrm{~g}$ plan$\mathrm{ta}^{-1}$, com produção máxima atingida aos $84 \mathrm{DAE}$, ocasião em que as plantas se apresentaram senescentes. Estes dados podem ser decorrentes do acréscimo da estrutura reprodutiva à parte aérea, a qual incrementa a massa da parte aérea da planta.

A TCA dos biótipos apresentou comportamento semelhante à variável $A F$, com ajuste dos dados ao modelo quadrático (Figura 3). A partir de 42 DAE, a TCA não diferiu das demais avaliações, à exceção dos $54 \mathrm{DAE}$, o qual foi superior a $42 \mathrm{DA}$ E. O máximo crescimento dos biótipos foi observado aos $64 \mathrm{DAE}$, o que pode ter decorrido do início do processo reprodutivo. A partir desse momento, o incremento da parte reprodutiva aumentou a massa, porém a relação da massa seca da parte aérea em relação ao tempo decresceu.

Por sua vez, a RAFMAS de plantas de $C$. difformis decresceu com o tempo (Figura 4). A cada acréscimo de um dia no tempo após a emergência, verificou-se redução de $0,0023 \mathrm{dm}^{2} \mathrm{~g}^{-1}$, sendo a menor RAFMAS observada ao final do período de avaliação. Estes resultados demonstram a relação entre AF e MPAS com o decorrer do ciclo da cultura, onde ambas aumentam devido a expansão da AF e também a contribuição da estrutura reprodutiva no incremento da MPAS. 
DAL MAGRO, T. et al. Características fisiológicas e de desenvolvimento...

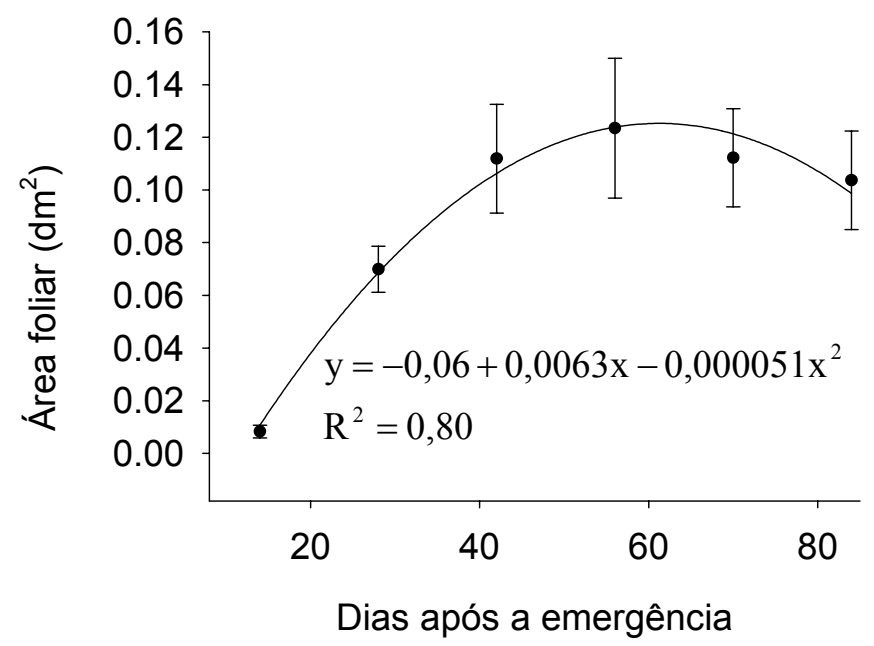

FIGURA 1 - Área foliar de plantas de Cyperus difformis resistente e suscetível ao herbicida pyrazosulfuronethyl. UFPel, Capão do Leão - RS, 2006/07. (Pontos representam os valores médios das repetições e as barras verticais os intervalos de confiança referentes ao desvio padrão da média).

Os resultados demonstram não haver perdas do biótipo resistente aos herbicidas inibidores da enzima ALS, quanto a características de desenvolvimento. Esses resultados poderão decorrer da capacidade volumétrica dos recipientes utilizados, limitando o crescimento da parte aérea e do sistema radicular aonde, se conduzidos em condições distintas, poderia gerar resultados diferentes. Porém, resultados semelhantes aos do presente trabalho foram observados para Bidens subalternans (Lamego, 2008), Kochia scoparia (Thompson et al., 1994) e S. montevidensis (Rampelotti et al., 2003), em que os biótipos resistentes e suscetíveis aos herbicidas inibidores da enzima ALS apresentaram características de desenvolvimento idênticas demonstrando que, nas condições que foram conduzidas o experimento, a semelhança entre os biótipos assemelha-se aos dados da literatura.

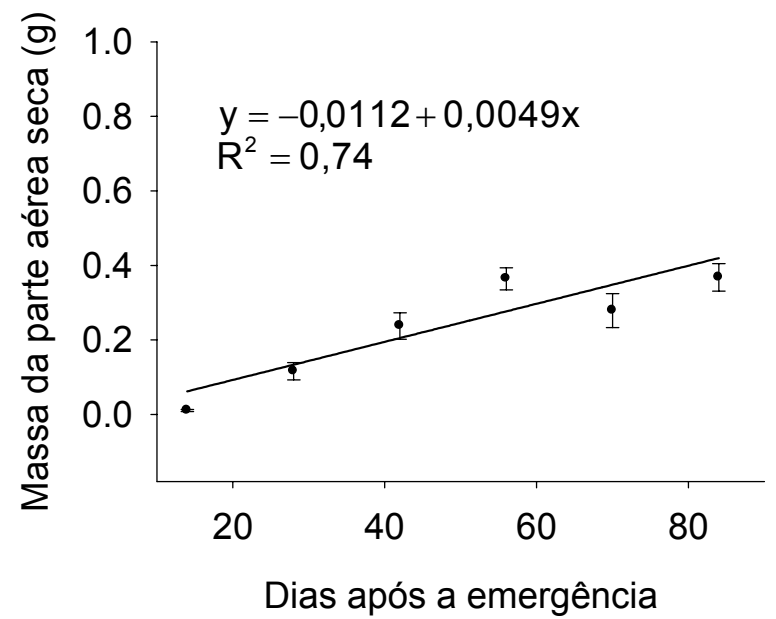

FIGURA 2 - Massa da parte aérea seca (g por planta) de plantas de Cyperus difformis resistente e suscetível ao herbicida pyrazosulfuron-ethyl. UFPel, Capão do Leão - RS, 2006/07. (Os pontos representam os valores médios das repetições e as barras verticais os intervalos de confiança referentes ao desvio padrão da média). 
DAL MAGRO, T. et al. Características fisiológicas e de desenvolvimento...

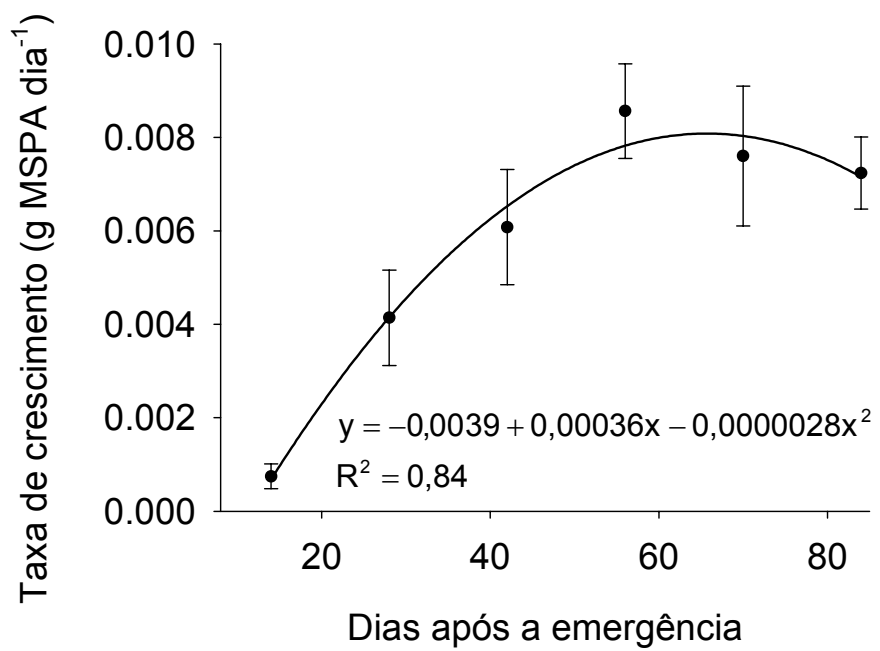

FIGURA 3 - Taxa de crescimento absoluto de plantas de Cyperus difformis resistente e suscetível ao herbicida pyrazosulfuron-ethyl. UFPel, Capão do Leão - RS, 2006/07. (Os pontos representam os valores médios das repetições e as barras verticais os intervalos de confiança referentes ao desvio padrão da média).

\section{Experimento II}

Nas avaliações realizadas aos 7 e 14 DAT, não houve significância estatística dos fatores testa- dos (Tabelas 1 a 3), à exceção da taxa de fluxo de gases pelos estômatos para o fator herbicida, avaliada aos 7 DAT (Tabela 2), e do $\mathrm{CO}_{2}$ consumido

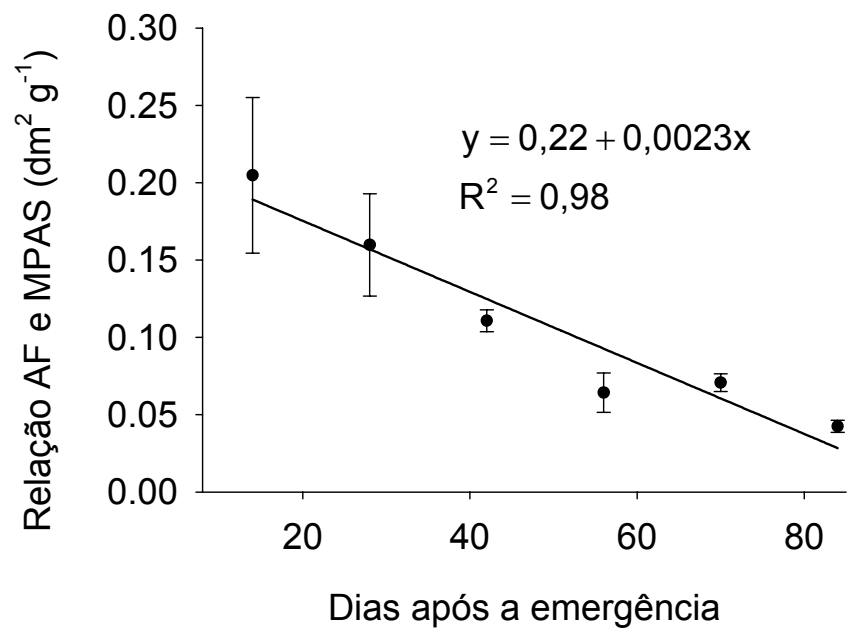

FIGURA 4 - Relação área foliar (AF) e massa da parte aérea seca (MPAS) de plantas de Cyperus difformis resistente e suscetível ao herbicida pyrazosulfuron-ethyl. UFPel, Capão do Leão - RS, 2006/07. (Os pontos representam os valores médios das repetições e as barras verticais os intervalos de confiança referentes ao desvio padrão da média). 
DAL MAGRO, T. et al. Características fisiológicas e de desenvolvimento...

TABELA $1-\mathrm{CO}_{2}$ consumido $(\Delta \mathrm{C})$, concentração de $\mathrm{CO}_{2}$ sub-estomático $(\mathrm{C})$, taxa transpiratória $(\mathrm{E})$, condutância estomática de vapores de água (Gs), taxa fotossintética (A) e eficiência do uso da água (EUA) de biótipos de Cyperus difformis resistente e suscetível ao herbicida pyrazosulfuron-ethyl avaliados aos 7 e 14 dias após a aplicação (DAT). UFV, Viçosa - MG, 2008.

\begin{tabular}{lcc}
\hline Variáveis & 7 DAT & 14 DAT \\
\hline$\Delta \mathrm{C}\left(\mu \mathrm{mol} \mathrm{mol}^{-1}\right)$ & 7,5 & 11,3 \\
$\mathrm{C}\left(\mu \mathrm{mol} \mathrm{mol}{ }^{-1}\right)$ & 351,1 & 328,0 \\
$\mathrm{E}\left(\mathrm{mol} \mathrm{H}_{2} \mathrm{O} \mathrm{m}^{-2} \mathrm{~s}^{-1}\right)$ & 2,5 & 1,7 \\
$\mathrm{Gs}\left(\mathrm{mol} \mathrm{m}^{-1} \mathrm{~s}^{-1}\right)$ & 0,5 & 0,3 \\
$\mathrm{~A}\left(\mu \mathrm{mol} \mathrm{m} \mathrm{s}^{-1}\right)$ & 3,0 & 3,4 \\
EUA $\left(\mathrm{mol} \mathrm{CO}_{2} \mathrm{~mol} \mathrm{H}_{2} \mathrm{O}^{-1}\right)$ & 1,1 & 2,1 \\
\hline
\end{tabular}

A taxa de fluxo de gases pelos estômatos, avaliada aos 7 DAT, apresentou diferença estatística entre tratamentos, sendo maior no tratamento que recebeu 0 herbicida pyrazosulfuron-ethyl
(Tabela 2). $\mathrm{O} \quad \mathrm{CO}_{2}$ consumido, avaliado aos 14 DAT, diferiu entre os biótipos, quando o biótipo resistente apresentou maior consumo de $\mathrm{CO}_{2}$ comparativamente ao suscetível (Tabela 3 ).

TABELA 2 - Taxa de fluxo de gases pelos estômatos $(U)$ de biótipos de Cyperus difformis resistente e suscetível ao herbicida pyrazosulfuron-ethyl avaliada aos 7 dias após a aplicação. UFV, Viçosa - MG, 2008.

\begin{tabular}{lc}
\hline Tratamentos & $\mathrm{U}\left(\mu \mathrm{mol} \mathrm{s}^{-1}\right)$ \\
\hline Testemunha & 199,1 \\
Pyrazosulfuron-ethyl & 200,7 \\
\hline
\end{tabular}

* Significativo pelo teste $t(p \leq 0,05)$.

TABELA $3-\mathrm{CO}_{2}$ consumido $(\Delta \mathrm{C})$ por biótipos de Cyperus difformis resistente e suscetível ao herbicida pyrazosulfuron-ethyl, avaliado 14 dias após a aplicação. UFV, Viçosa - MG, 2008.

\begin{tabular}{lc}
\hline Biótipos & $\Delta \mathrm{C}\left(\mu \mathrm{mol} \mathrm{mol}{ }^{-1}\right)$ \\
\hline Resistente & 11,5 \\
Suscetível & 11,0 \\
\hline
\end{tabular}

* Significativo pelo teste $t(p \leq 0,05)$.

$\mathrm{Na}$ avaliação realizada aos 21 DAT, não houve significância estatística dos fatores testados, à exceção de $\mathrm{CO}_{2}$ consumido e da concentração de $\mathrm{CO}_{2}$ sub-estomático (Tabelas 4 e 5 ). $\mathrm{O} \mathrm{CO}_{2}$ consumido pelos biótipos de C. difformis, avaliado aos 21 DAT, diferiu entre a testemunha e as plantas trata- das com o herbicida pyrazosulfuron-ethyl, demonstrando maior consumo nas plantas que foram tratadas com o herbicida (Tabela 5). Por outro lado, a concentração de $\mathrm{CO}_{2}$ sub-estomático apresentou maior teor na testemunha.

TABELA 4 - Taxa de fluxo de gases pelos estômatos $(U)$, taxa transpiratória (E), condutância estomática de vapores de água (Gs), taxa fotossintética (A), eficiência do uso da água (EUA) de biótipos de Cyperus difformis resistente e suscetível ao herbicida pyrazosulfuron-ethyl, avaliados 21 dias após a aplicação do tratamento (DAT). UFV, Viçosa - MG, 2008.

\begin{tabular}{lc}
\hline Variáveis & Médias \\
\hline$U\left(\mu \mathrm{mol} \mathrm{s}{ }^{-1}\right)$ & 199,9 \\
$E\left(\mathrm{~mol} \mathrm{H}_{2} \mathrm{O} \mathrm{m}^{-2} \mathrm{~s}^{-1}\right)$ & 1,6 \\
$\mathrm{Gs}\left(\mathrm{mol} \mathrm{m}^{-1} \mathrm{~s}^{-1}\right)$ & 0,2 \\
$\mathrm{~A}\left(\mu \mathrm{mol} \mathrm{m} \mathrm{s}^{-1}\right)$ & 2,6 \\
$\mathrm{EUA}\left(\mathrm{mol} \mathrm{CO}_{2} \mathrm{~mol} \mathrm{H}_{2} \mathrm{O}^{-1}\right)$ & 1,8 \\
\hline
\end{tabular}


TABELA $5-\mathrm{CO}_{2}$ consumido $(\Delta \mathrm{C})$ e concentração de $\mathrm{CO}_{2}$ sub-estomático $(\mathrm{C})$ de biótipos de Cyperus difformis resistente e suscetível ao herbicida pyrazosulfuron-ethyl, avaliados 21 dias após a aplicação. UFV, Viçosa MG, 2008.

\begin{tabular}{llc}
\hline Parâmetros & \multicolumn{1}{c}{ Tratamentos } & Médias \\
\hline$\Delta \mathrm{C}\left(\mu \mathrm{mol} \mathrm{mol}{ }^{-1}\right)$ & testemunha & $7,5^{*}$ \\
& pyrazosulfuron-ethyl & 9,0 \\
\hline $\mathrm{C}\left(\mu \mathrm{mol} \mathrm{mol}{ }^{-1}\right)$ & testemunha & 376,7 \\
& pyrazosulfuron-ethyl & 363,9 \\
\hline
\end{tabular}

* Significativo pelo teste $t(p \leq 0,05)$.

Para a variável MPAS houve interação dos fatores testados (Tabela 6). Para o biótipo resistente, a comparação entre tratamentos não apresentou diferença. Entretanto para o biótipo suscetível, a aplicação do herbicida reduziu a MPAS, diferindo da testemunha. Na comparação entre biótipos, houve diferença entre os mesmos apenas quando receberam o herbicida pyrazosulfuron-ethyl, com maior produção de MPAS para o biótipo resistente.

TABELA 6 - Massa da parte aérea seca (g por planta) de biótipos de Cyperus difformis resistente e suscetível ao herbicida pyrazosulfuron-ethyl, avaliada aos 28 dias após a aplicação. UFV, Viçosa - MG, 2008.

\begin{tabular}{lcc}
\hline \multirow{2}{*}{ Tratamentos } & \multicolumn{2}{c}{ Biótipos } \\
\cline { 2 - 3 } & Resistente & Suscetível \\
\hline Testemunha & ${ }^{n s} 0,64_{*}{ }^{\text {ns1 }}$ & \multirow{2}{*}{0,75} \\
Pyrazosulfuron-ethyl & 0,70 & 0,36 \\
\hline Média & & 0,42 \\
\hline C.V. $(\%)^{2}$ & & 17,40 \\
\hline
\end{tabular}

${ }^{1}$ Médias antecedidas, comparadas na coluna ou sucedidas, comparadas na linha; ${ }^{\text {ns }}$ ou * representam significativo e não significativo pelo teste $t(p \leq 0,05) .{ }^{2} \mathrm{C} . \mathrm{V} .=$ coeficiente de variação.

A maior taxa de fluxo de gases pelos estômatos $(U)$ no tratamento que recebeu o herbicida pyrazosulfuron-ethyl (Tabela 2) pode ser explicada pelo maior consumo de $\mathrm{CO}_{2}$ pelo biótipo resistente, pois o $U$ é a relação entre entrada de $\mathrm{CO}_{2}$ e saída de $\mathrm{O}_{2}$ na planta. Isto indica o quanto a planta está consumindo de $\mathrm{CO}_{2}$ para realização da fotossíntese, corroborando com a produção elevada de MPAS das plantas.

Esses resultados não corroboram a hipótese do estudo, na qual a resistência acarretaria perdas às funções fotossintéticas e de uso da água pelos biótipos. Entretanto, em geral, isso não ocorreu e os biótipos apresentaram características de uso da água semelhantes, corroborando os dados observados para as outras características biológicas estudadas, nas quais os biótipos apresentaram equivalência.
Com relação à produção de MPAS, foi possível observar que apenas o biótipo suscetível apresentou redução da mesma, sendo que do biótipo resistente apresentou produção equivalente à testemunha. Outra variável que apresentou diferença entre biótipos e pode estar relacionada positivamente com a maior produção de MPAS foi o consumo de $\mathrm{CO}_{2}$, sendo que o biótipo resistente apresentou maiores índices, justificando a maior produção de MPAS. Estes resultados contradizem os observados para azevém resistente a glyphosate, nos quais o biótipo resistente incorpora menos $\mathrm{CO}_{2} \mathrm{e}$ apresenta menor produção de MPAS (Concenço et al., 2007), podendo, desta forma, ser utilizada a seleção reversa como forma de manejo da espécie. 


\section{DAL MAGRO, T. et al. Características fisiológicas e de desenvolvimento...}

Este trabalho demonstrou que os dois biótipos de junquinho, resistente e suscetível ao herbicida pyrazosulfuron-ethyl, apresentam características fisiológicas e de desenvolvimento similar. Na literatura são relatados casos de alterações no desenvolvimento de biótipos de Amaranthus powelli resistente a herbicidas inibidores de ALS, os quais tiveram redução em seu crescimento e desenvolvimento decorrente da resistência (Tardif et al., 2006). Entretanto, em geral, essas alterações podem ocorrer em biótipos de plantas daninhas resistentes aos herbicidas inibidores de Fotossistema II (FSII), que apresentam mutações no gene psbA (proteína D1) com substituição do aminoácido serina por glicina na posição 264 , o qual codifica a quinona $\left(Q_{b}\right)$. Esta mutação, além de reduzir a afinidade da molécula herbicida com a $Q_{b}$, reduz o fluxo de elétrons no FSII, influenciando no processo fundamental para o crescimento e a sobrevivência das plantas. Dessa forma, o biótipo resistente apresentou redução na fixação de $\mathrm{CO}_{2} \mathrm{e}$, conseqüentemente, redução na produção de MPAS (Powles \& Holtum, 1994).

Outro ponto a considerar é que o estudo foi conduzido com apenas dois biótipos de C. difformis, um resistente e um suscetível, que apresentaram características de desenvolvimento similar, demonstrando que novos estudos podem ser desenvolvidos com esta espécie, abrangendo maior número de biótipos. Os resultados desses experimentos restringem a adoção de medidas de manejo como seleção reversa, sendo que a sua adoção implicaria no aumento na população dos dois biótipos. Com isso, medidas de controle que evitem a proliferação da espécie devem ser avaliadas, considerando o manejo integrado da espécie.

\section{CONCLUSÕES}

Os biótipos resistente e suscetível de $\mathrm{Cy}$ perus difformis ao herbicida pyrazosulfuron-ethyl apresentam características fisiológicas e de desenvolvimento semelhantes.

\section{REFERÊNCIAS}

1. AGOSTINETTO, D. et al. Competitividade relativa entre cultivares de arroz irrigado e biótipo de capim-arroz (Echinochloa spp.). Planta Daninha, v.26, n.4, p.757-766, 2008.

2. ALCOCER-RUTHLING, M.; THILL, D.C.; SHAFII, B. Differential competitiveness of sulfonylurea-resistant and-susceptible prickly lettuce (Lactuca serriola). Weed Technology, v.6, n.1, p.303-309, 1992.

3. CONARD, S.G.; RADOSEVICH, S.R. Ecological fitness of Senecio vulgaris and Amaranthus retroflexus biotypes susceptible or resistant to atrazine. Journal Applied Ecology, v.17, n.1, p.171-177, 1979.

4. CONCENÇO, G. et al. Uso da água em biótipos de azevém (Lolium multiflorum) em condição de competição. Planta Daninha, v.25, n.3, p.449-455, 2007.

5. FLECK, N.G. et al. Competitividade relativa entre cultivares de arroz irrigado e biótipo de arroz-vermelho. Planta Daninha, v.26, n.1, p.101-111, 2008

6. GRAY, J.A.; STOLTENBERG, D.E.; BALKE, N.E. Productivity and intranspecific competitive ability of a velvetleaf (Abutilon theophrasti) biotype resistant to atrazine. Weed Science, v.43, n.4, p.619-626, 1995

7. LAMEGO, F.P. Elucidação do mecanismo de resistência aos herbicidas inibidores de ALS na espécie poliplóide Bidens subalternans DC. 2008. 152f. Tese (Doutorado em Fitotecnia)- Universidade Federal do Rio Grande do Sul, Porto Alegre.

8. NOLDIN, J.A.; EBERHARDT, D.S.; RAMPELOTTI, F. Cyperus difformis L. resistente a herbicidas inibidores da ALS em Santa Catarina. In: CONGRESSO BRASILEIRO DA CIÊNCIA DAS PLANTAS DANINHAS, 23., 2002, Gramado. Anais... Londrina: SBCPD, p.198, 2002.

9. PARKS, R.J. et al. Herbicide susceptibility and biological fitness of triazine-resistant and susceptible common lambsquarters (Chenopodium album). Weed Science, v.44, n.3, p.517-522, 1996.

10. POWLES, S.B.; HOLTUM, J.A.M. Herbicides resistance in plants: biology and biochemistry. New York: CCR Press, 1994. 353p.

11. RADOSEVICH, S.; HOLT, J.; GHERSA, C. Weed ecology: implications for management. 2.ed. New York: Willey, 1997. 589p. Cap. 6: Physiological aspects of competition.

12. RAMPELOTTI, F.T. et al. Análise de crescimento de ecótipos de sagitária (Sagittaria montevidensis) resistente e suscetível a herbicidas inibidores da ALS. In: CONGRESSO BRASILEIRO DE ARROZ IRRIGADO, CONGRESSO BRASILEIRO DE ARROZ IRRIGADO, 3., 2003, Balneário Camboriú, SC. Anais... Itajaí: EPAGRI, p.483-485, 2003.

13. TARDIF, F.J.; RAJCAN, I.; COSTEA, M. A mutation in the herbicide target side acetohydroxyacid synthase produces morphological and structural alterations and reduces fitness in Amaranthus powellii. New Phytologist, v.169, n.2, p.251-264, 2006.

14. THOMPSON, C.R.; THILL, D.C.; SHAFII, B. Growth and competitiveness of sulfonylurea-resistant and susceptible kochia (Kochia scoparia). Weed Science, v.42, n.2, p.172-179, 1994.

15. VARGAS, L. et al. Alteração das características biológicas dos biótipos de azevém (Lolium multiflorum) ocasionada pela resistência ao herbicida glyphosate. Planta Daninha, v.23, n.1, p.153-160, 2005.

Recebido em 28/01/2010 Aceito em 29/11/2011 